vol. 26 - $n^{\circ} 2 \mid 2010$

Numéro ouvert

\title{
Du diplomate à l'expatrié. Les migrations chinoises en Suisse
}

From diplomat to expatriate. Chinese migration to Switzerland

Del diplomático al expatriado. Las migraciones chinas en Suiza

\section{Marylène Lieber}

\section{(2) OpenEdition}

Journals

Édition électronique

URL : https://journals.openedition.org/remi/5163

DOI : 10.4000/remi.5163

ISSN : $1777-5418$

Éditeur

Université de Poitiers

Édition imprimée

Date de publication : 1 septembre 2010

Pagination : 191-214

ISBN : 978-2-911627-55-2

ISSN : 0765-0752

Référence électronique

Marylène Lieber, « Du diplomate à l'expatrié. Les migrations chinoises en Suisse », Revue européenne

des migrations internationales [En ligne], vol. 26 - n² | 2010, mis en ligne le 01 septembre 2013,

consulté le 14 avril 2022. URL : http://journals.openedition.org/remi/5163 ; DOI : https://doi.org/

10.4000/remi.5163 


\section{Note de recherche}

\section{Du diplomate à l'expatrié Les migrations chinoises en Suisse}

\section{Marylène LIEBER}

T es migrations chinoises en Suisse n'ont pas fait l'objet de recherches appro-

fondies, contrairement à plusieurs autres pays d'Europe, comme la France, la Hollande ou la Grande-Bretagne (Live, 1992 ; Pieke, 1992 ; Vaughan 1992). On ne sait donc pratiquement rien sur les Chinois de Suisse, sinon que, à l'inverse de ceux installés dans les pays voisins, leur nombre est marginal et qu'ils ne sont pas réellement visibles ${ }^{1}$

Aucune ville suisse n'a de Chinatown ou de quartier présentant une forme de concentration résidentielle, comme on peut en rencontrer à Paris ou à Londres, pas plus qu'il n'existe de presse spécialisée en mandarin, ou d'associations représentatives qui pèseraient réellement dans la vie politique locale - ce qui, cela dit, est vrai également pour d'autres groupes de migrants vivant en Suisse. Néanmoins, cette population connaît aujourd'hui de véritables bouleversements, puisque dans le sillage des autres pays d'Europe, elle a vu son nombre officiel presque décupler durant les vingt dernières années, passant de 846 en 1988 à 7612 en 2008 . Cette croissance est allée de paire, durant la dernière décennie, avec un essor de formes d'organisations liées à la dimension ethnique, que ce soient des entreprises, tels que des restaurants ou des centres de médecine chinoise, ou des associations dont le but est de promouvoir les intérêts de la population d'origine chinoise, et dont le nombre est de plus en plus important (Lieber, 2010).

* Docteure en sociologie, Chercheuse post-doc, MAPS, Université de Neuchâtel, Suisse et Chercheuse invitée, CEFC, antenne de Taipei, Shidong road, lane 91, alley 26, no 3, Taipei 111, Taiwan (ROC) ; marylene.lieber@gmail.com Je tiens à remercier Janine Dahinden, Luca Gabbiani, Ellen Hertz, Florence Lévy, Hélène Martin et Ionela Vlase pour leurs relectures attentives et leurs critiques constructives des premières versions de ce texte.

1 Cet article est issu de la recherche financée par le Fonds national suisse de la recherche scientifique (FNS), "Migrations chinoises en Suisse : structures et dynamiques des réseaux », dirigée par la professeure Ellen Hertz de l'institut d'ethnologie de l'Université de Neuchâtel. Le travail de terrain a été effectué par Florence Lévy et moi-même et est composé d'observations dans les lieux où les ressortissants chinois se regroupent, ainsi que quatre-vingt entretiens qualitatifs. Les données statistiques ont été traitées par les membres du Forum suisse pour l'étude des migrations et de la population (SFM) à l'Université de Neuchâtel : Marco Pecoraro et Rosita Fibbi. 
Face à ce manque d'informations et à ces mutations récentes, cet article entend tout simplement se demander qui sont les Chinois de Suisse. Et si cette question peut paraître simple, de prime abord, elle est en réalité fort complexe, puisque la catégorie « Chinois » recoupe des registres variés et dépasse la seule identité nationale (Kent, 2003). L'histoire politique et économique de la Chine est en effet telle qu'il existe deux Chine depuis la création en 1949 de la République populaire de Chine et l'exil du gouvernement nationaliste sur l'île de Taïwan ${ }^{2}$. Par ailleurs, il existe une importante diaspora ${ }^{3}$, notamment en Asie du Sud-Est, dont les descendants sont considérés et se considèrent comme Chinois, et qui ont pour une partie immigré à leur tour en Europe ou aux ÉtatsUnis.

Qui plus est, il y a une distinction sémiotique en mandarin que la traduction française ne peut souligner. Le terme « Chinois » peut se dire de plusieurs façons, qui sont utilisées selon les contextes pour marquer des distinctions proprement politiques. Ainsi, si les Chinois de Chine populaire se qualifient le plus souvent de zhongguoren, c'est-à-dire de personnes du pays du milieu, les Chinois de Singapour ou de Taïwan, se qualifient souvent de huaren, ce qui peut se traduire par personnes appartenant à l'aire culturelle chinoise. L'usage de ce second terme permet d'insister sur les liens avec la culture chinoise, mais de se distinguer de la Chine communiste. Pour autant, dans d'autres contextes, le terme huaren peut être utilisé à des fins idéologiques afin de rallier au régime de Pékin tous les Chinois du monde, quelle que soit leur nationalité (Nyiri, 1999). Réciproquement, certains ressortissants taïwanais revendiquent leur seule identité taïwanaise (voir par exemple pour les États-Unis, Zhou et Chiang, 2009) ou à l'inverse se qualifient de zhongguoren.

Il existe donc un véritable problème de fond lorsqu'on veut parler des migrations chinoises, puisque le sentiment d'appartenance à la catégorie « Chinois » recoupe différentes interprétations qui se rattachent à des registres divers, comme l'ethnie, la culture ou la nationalité (Ang, 1998). De fait, les Tibétains sont des ressortissants de Chine populaire, mais ne se considèrent pas comme « Chinois ». En revanche, pour des raisons diploma-

2 Après sa défaite face aux communistes en 1949, le gouvernement nationaliste de Tchang Kaï-Chek s'est réfugié sur l'île de Taiwan où ses partisans du Guomindang ont continué à se poser comme seuls représentants de « la » Chine. C'est ce gouvernement, la République de Chine (créée sur le continent en 1911), qui a été reconnu par les Nations Unies jusqu'en 1971, avant d'être évincé au profit de la République populaire de Chine. Si la République populaire de Chine exige la reconnaissance d'une seule Chine, il existe dans les faits, « deux Chines ». La République de Chine (Taiwan) n'est reconnue comme un pays à part entière que par vingt-trois nations, mais c'est un État effectif dans la mesure où elle a son propre gouvernement et ses propres institutions. Dans cet article, je me réfère à la République de Chine en parlant de Taiwan ou du gouvernement nationaliste, tandis que je parle de la République populaire de Chine, en parlant de Chine populaire, de Chine continentale ou du gouvernement communiste.

3 On estime que la diaspora chinoise, qui s'est constituée au fil des siècles, regroupe actuellement entre trente à cinquante millions de personnes dans le monde. Les pays traditionnels d'immigrations chinoises sont les pays du Sud-Est asiatique et les pays d'Amérique du Nord. Si l'Europe a également connu des flux en provenance de Chine dès la moitié du XIXe siècle, celle-ci ne concentre aujourd'hui que $6 \%$ des effectifs mondiaux (contre environ $80 \%$ en Asie et $14 \%$ en Amérique) (Ma, 2003). 
tiques, les institutions suisses, elles, les considèrent comme Chinois ${ }^{4}$. Ceux qu'on qualifie souvent de « Chinois ethniques », les Chinois du Vietnam, de Singapour ou d'Indonésie, se considèrent comme Chinois, mais n'ont jamais vécu en Chine et n'ont pas la nationalité chinoise. D'ailleurs, en Suisse, les autres Chinois les qualifient de huaqiao, terme usuellement utilisé pour parler des Chinois d'Outre-mer qui ont la nationalité chinoise (Wang, 1992), ce qui n'est justement pas leur cas ${ }^{5}$. Ils ne sont donc pas comptabilisés comme Chinois par les institutions suisses. Les Taïwanais, quant à eux, se définissent comme Chinois ou Taïwanais, selon leur appartenance politique et leur adhésion à une reconnaissance de Taïwan comme d'un pays à part entière. La Suisse les comptabilise séparément. Pourtant, lorsqu'on participe à une fête de Nouvel An chinois ou qu'on assiste à une classe de mandarin dans une des écoles du week-end, on est amené à côtoyer tout autant des Chinois d'Asie du Sud-Est, que des Taïwanais ou des gens de Pékin. D'ailleurs, beaucoup d'entre eux ont la nationalité suisse, qu'ils l'aient acquise par naturalisation ou par mariage. On ne retrouve, en revanche, aucun Tibétain, qui sont pour la plupart des ressortissants chinois! Un véritable casse-tête !

Alors, dans ces circonstances, qui sont les Chinois de Suisse et comment peut-on caractériser leur migration ? Cet article présente tout d'abord les statistiques officielles suisses, qui donnent à voir une figure de migrant chinois extrêmement qualifié, avant d'adopter une perspective qui embrasse la complexité de la catégorie « Chinois » et reflète la diversité des personnes qui se considèrent comme telles. En mettant l'accent sur le processus de construction identitaire, qui se reconfigure dans la migration en fonction des contextes locaux (Ma, 2003), les statistiques officielles se verront en effet complétées par des données qualitatives sur les spécificités des Chinois de Suisse et sur les mutations récentes connues par cette population. Ainsi, la seconde partie propose une typologie des diverses catégories de Chinois, en fonction du cadre et de l'époque de la migration, qui souligne également le haut niveau socio-économique des Chinois de Suisse, et ce, à toutes les époques. La troisième partie, quant à elle, se penche sur l'évolution de leurs modes d'organisation, qui laisse penser qu'on observe, comme ailleurs, une revitalisation des liens des Chinois vivant à l'étranger avec la République populaire de Chine (Hong, 2005).

4 Depuis 2000, en effet, ils ne sont plus comptabilisés séparément. Qui plus est, à la suite d'une décision, en 2004, de la Commission de recours en matière d'asile (CRA), les autorités suisses considèrent comme ayant la nationalité chinoise des Tibétains ayant vécu au Népal ou en Inde.

5 Il existe un vrai débat sur la terminologie appropriée pour parler des Chinois vivant à l'étranger. Wang (1992) a suggéré de n'utiliser le terme de huaqiao (Chinois temporairement à l'étranger) que pour les seuls ressortissants chinois, même s'il a souvent été utilisé dans le sens commun pour parler de tous les Chinois de l'étranger, quelle que soit leur nationalité, et ce, pour la simple raison qu'il a été forgé par le gouvernement chinois dès le XIXe siècle pour parler des citoyens chinois vivant en dehors du pays. Ainsi, il estime que pour les « Chinois » vivant à l'étranger, s'ils ont une autre nationalité, le terme de huayi (descendants de Chinois) ou haiwaihuaren (Chinois vivant à l'étranger) doit leur être préféré. En anglais, on parle alors souvent de Chinois « ethniques » (ethnic Chinese). 


\section{CE QUE NOUS DISENT LES STATISTIQUES SUISSES}

Les deux principales sources de données statistiques dont on dispose pour mieux connaître le profil des Chinois de Suisse sont les recensements fédéraux de la population disponibles depuis 1970 et la Statistique de la population résidante de nationalité étrangère, pour la dernière décennie ${ }^{6}$. De nature parcellaire, on l'a vu, ces données permettent de mettre en évidence certaines spécificités de la population chinoise résidant en Suisse.

À la fin décembre 2008, l'Office fédéral des migrations (ODM) comptait 7612 ressortissants de la République populaire de Chine résidant sur le territoire helvétique ${ }^{7}$. Ces chiffres officiels peuvent être mis en parallèle avec d'autres estimations, comme les appréciations émanant de Chinois résidant en Suisse, eux-mêmes, ou comme les statistiques tenues par le gouvernement taïwanais, qui comptabilisent les «Chinois » du monde, quelle que soit leur nationalité. Ainsi, la commission taïwanaise des affaires des expatriés $(\mathrm{OCAC})^{8}$ avance le nombre de 14000 Chinois vivant en Suisse pour l'année 2007 entendus ici comme des personnes de "culture chinoise » (huaren) (OCAC, 2007) quand certains journalistes chinois parlent, eux, de 20000 à 30000 personnes ${ }^{9}$.

Quoi qu'il en soit, les Chinois ne représentent qu'une petite proportion de la population étrangère (entre 0,45 et $1,8 \%$ selon les sources $)^{10}$. La Suisse compte donc une immigration chinoise relativement peu importante et qui paraît marginale en regard de celle des pays voisins ${ }^{11}$. Cela dit, proportionnellement, cette population a augmenté de façon significative à partir des années 1990, avant de se stabiliser à partir du début des années 2000 (Figure 1). Cet accroissement se vérifie également dans les évaluations taïwanaises (OCAC, 2007) ${ }^{12}$.

6 Les données statistiques de cette partie ont été traitées par Marco Pecoraro et Rosita Fibbi (2008), du Forum suisse pour l'étude des migrations et de la population (SFM) à l'Université de Neuchâtel. Elles sont également tirées du mémoire de maîtrise de Pascal Rocha da Silva (2007), en démographie économique et sociale de l'Université de Genève. Pour plus d'informations sur les statistiques disponibles en Suisse, voir également : http://www.bfs.admin.ch/bfs/portal/fr/ index/themen/01/22/publ/ausl/presentation.Document.116849.pdf (consulté le 12 février 2009).

7 Ces chiffres correspondent aux ressortissants de la République populaire de Chine, titulaires d'un permis de séjour et d'établissement.

8 Overseas Compatriot Affairs Commission. À noter que cette commission s'appelait initialement Overseas Chinese Affairs Commission et que ce changement de nom est le reflet du débat politique quant au statut de l'île (voir par exemple sur ce second sujet : Brown, 2004).

$9 \mathrm{http} / / \mathrm{www}$. swissinfo.ch/chi/news/shortnews/detail.html?siteSect=106\&sid=9867340\&cKey=12 $24498478000 \&$ ty $=$ st.

10 La Suisse comptait une population étrangère de 1669700 personnes en 2008, dont 86,5\% de ressortissants d'un pays d'Europe. http://www.bfs.admin.ch/bfs/portal/fr/index/themen/01/07/ blank/key/01/01.html

11 À titre d'exemple, l'Italie comptait en 2006114165 ressortissants de République de Chine populaire, l'Espagne 85745 en 2005 et la Grande-Bretagne 67930 en 2004 (Pecoraro et Fibbi, 2008).

12 Selon les statistiques du gouvernement suisse, le nombre de ressortissants taïwanais est resté stable, environ 400 personnes. 
Figure 1 : Les ressortissants de République populaire de Chine en Suisse

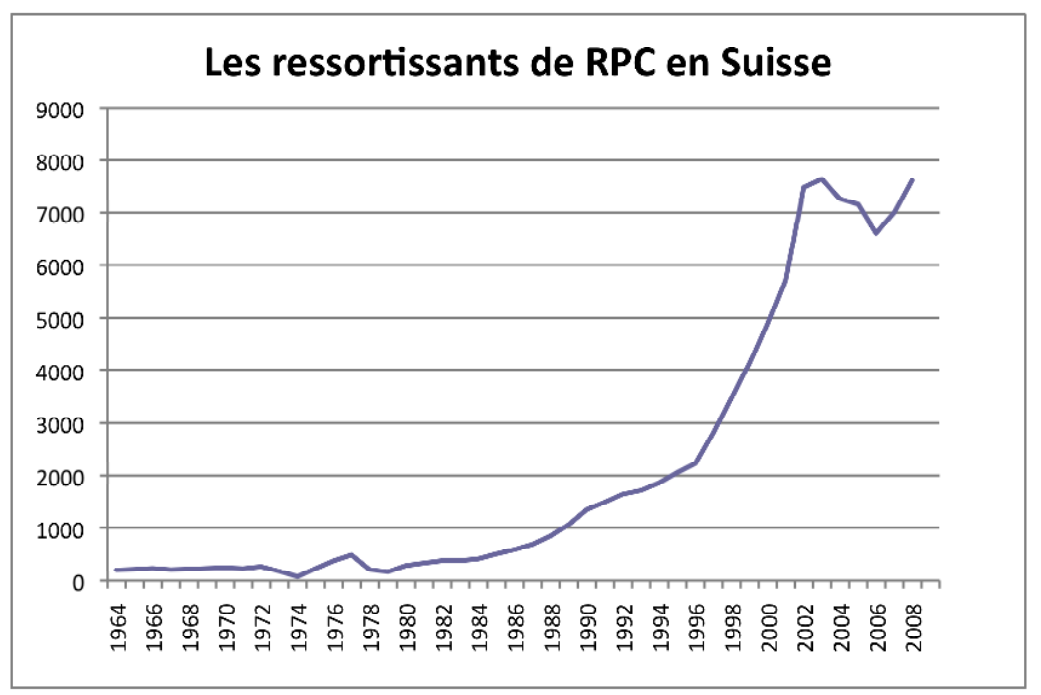

Source : OFS/RCE 2008

Deux caractéristiques majeures ressortent des statistiques suisses et soulignent le haut niveau de qualification des Chinois qui résident dans ce pays. Tout d'abord, c'est la forte proportion d'étudiants ${ }^{13}$. Les ressortissants de République populaire de Chine sont une population plutôt jeune (deux tiers ont entre vingt et trente-neuf ans), dont une grande majorité est arrivée relativement récemment (plus de deux tiers de ces personnes se sont installés depuis moins de six ans) et qui suit une formation. Le taux de personnes en formation est en effet plus important pour les Chinois que celui d'autres populations. $65 \%$ des Chinois non actifs sont en formation, contre 13,9\% des Suisses. Inversement, la proportion des Chinois âgés de vingt et vingt-quatre ans qui ne sont pas en train de suivre une formation n'atteint pas la moitié de celle des Suisses du même âge (Rocha, 2007).

Cette caractéristique « estudiantine » de la population chinoise en Suisse se reflète dans sa répartition sur le territoire suisse. Elle se concentre dans les grands centres urbains et leur périphérie, et ce, notamment, dans les lieux où il y a des écoles privées (comme le Valais, voire Lucerne) ou des universités renommées (comme Genève ou Bâle, pour la pharmacie et la chimie) et des écoles polytechniques (comme à Lausanne et à Zurich). Les cantons de Zurich, Genève et Vaud cumulent ainsi $50 \%$ des Chinois résidant en Suisse.

Deuxième caractéristique, la Suisse compte une proportion importante de cadres chinois. Dans un article récent, Zhang Guochu (2003) estime que le flux des migrations

13 Caractéristique qui explique en partie le taux important de femmes (54 \% en 2005). Cette féminisation s'explique également par la prépondérance des mariages binationaux avec des ressortissants suisses. 
internationales de Chinois hautement qualifiés a pour destination l'Europe et les ÉtatsUnis. Cette tendance est également vraie pour la Suisse. En 2000, en comparaison de la population active de nationalité suisse, les ressortissants chinois avaient achevé un niveau d'étude supérieur. La majorité des Suisses ont en effet un diplôme secondaire (58 \%), alors que la population active chinoise résidant en Suisse a soit terminé un doctorat $(51,4 \%)$, ou à l'inverse n'a pas de formation $(9,8 \%)$. Cette situation contrastée découle sans doute de la politique sélective d'immigration qui favorise le personnel hautement qualifié (Piguet, 2004). On compte ainsi, en 2000, trois fois plus de cadres supérieurs dans la population active chinoise que chez les actifs suisses (resp. $28 \%$ et 10,9\%), pour trois fois moins d'ouvriers qualifiés (resp. 3,1\% et 10,3\%) et deux fois moins d'employés (resp. 13,5\% pour $27,3 \%$ ). Si, le secteur d'activité qui regroupe la plus grande proportion de la population active chinoise reste certes celui de la restauration, il ne concerne toutefois qu'un quart des ressortissants chinois en Suisse (Pecoraro et Fibbi, 2008).

Deux tendances générales se dégagent donc de ces données. Tout d'abord, les Chinois de Suisse n'ont pas le même profil socio-économique que les groupes majoritaires dans les autres pays européens, comme l'Italie, la Hollande ou la France, où l'on trouve une chaîne migratoire entrepreneuriale provenant du sud du Zhejiang ou du Fujian (deux provinces rurales du Sud-Est de la Chine et régions traditionnelles d'émigration) (Campani et al., 1994 ; Pina-Guérassimoff, 2003 ; Skeldon, 2004) ${ }^{14}$. L'immigration chinoise en Suisse est donc principalement composée de personnes qualifiées et elle est très éloignée de l'image d'une immigration commerçante regroupée en communauté et coupée de la société d'accueil.

Pour autant, on peut considérer que le cas suisse s'inscrit dans les évolutions observées récemment au sujet des migrations chinoises, puisque depuis le lancement de la politique chinoise de « réforme et ouverture » dès la fin des années 1970, les mouvements migratoires des ressortissants de Chine populaire vers l'Europe se sont complexifiés de façon non négligeable ${ }^{15}$ et que de plus en plus de personnes qualifiées sont arrivées en Europe (et continuent de le faire), que ce soit pour poursuivre une formation, travailler pour des entreprises locales et dans de grands groupes internationaux, ou comme représentants d'entreprises chinoises implantées à l'étranger (Beltran, 2004 ; Guérassimoff, 2005 ; Zhang, 2003). Il faut toutefois souligner qu'en regard de la forte augmentation du nombre des étudiants chinois en Europe, la croissance constatée en Suisse est relativement faible ${ }^{16}$.

14 Les statistiques suisses ne fournissent pas d'information quant à la provenance régionale des ressortissants de la République populaire de Chine. Toutefois, les caractéristiques de cette population - beaucoup d'étudiants et de cols-blancs - permettent d'affirmer qu'il s'agit de personnes venant de toute la Chine et non pas de migrations en provenance d'une région en particulier.

15 Dès la fin des années 1990, ils augmentent drastiquement et connaissent une forte mobilité intraeuropéenne (Benton et Pieke, 1998 ; Nyiri et Saveliev, 2002). On constate, de plus, une diversification, que ce soit en termes d'origines régionales, de profils sociaux ou de pays de destination (Béja, 2001 ; Ma Mung, 2002). Ainsi, ce ne sont plus les seuls Chinois du Sud qui s'expatrient et on assiste à une augmentation significative des migrations, légales et illégales, vers de nouveaux pays d'Europe centrale et d'Europe du Sud.

16 En Grande-Bretagne par exemple, les Chinois de République populaire représentent le groupe le plus important des étudiants étrangers, tandis qu'en Hollande, leur nombre a augmenté de plus de $50 \%$ entre 2000 et 2001 (Laczko, 2003). 


\section{DU DIPLOMATE À L'EXPATRIÉ. UNE TYPOLOGIE DES CHINOIS DE SUISSE}

La migration chinoise en Suisse étant une migration de personnes hautement qualifiées, il convient maintenant de se demander qui sont ces migrants qualifiés. La démarche qualitative permet de mettre en évidence que derrière ce terme se cache une population fort variée. Sans prétendre parler de flux, tant le nombre de personnes concernées est faible, il importe de souligner que ce ne sont pas les mêmes types de populations chinoises qui sont arrivées en Suisse selon les époques et les contextes politiques. Cette seconde partie propose donc une typologie qui constitue une construction non exhaustive et discutable, mais qui est utile pour montrer la diversité des profils des Chinois de Suisse et pour souligner la structuration de ce groupe de migrants selon le critère des catégories socioprofessionnelles plutôt que celui des provenances géographiques - caractéristique qui relève du contexte sociopolitique et de la nature singulière des migrations de personnes hautement qualifiées.

Jusque dans les années 1970, les Chinois de Suisse étaient peu nombreux et extrêmement qualifiés, souvent des diplomates, des fonctionnaires internationaux ou des personnes issues de familles aisées. Dès la fin des années 1970, on observe une première rupture avec l'arrivée d'un contingent de Chinois du Sud-Est asiatique. Ceux-ci se verront à leur tour concurrencés, dès la fin des années 1990, par les Chinois qualifiés de Chine populaire - qu'ils soient expatriés ou étudiants.

\section{Les premières générations de Chinois hautement qualifiés}

Il n’y a pas eu en Suisse d'arrivée importante de personnes en provenance de Chine, qui permette d'expliquer la formation d'une " communauté chinoise », comme c'est le cas ailleurs en Europe ${ }^{17}$. Au contraire, du début du XXe siècle, jusqu'à la fin des années 1970, ce sont des petits nombres de personnes de catégories diverses qui se sont succédés.

C'est surtout le caractère international de Genève qui explique la présence, dès les années 1920, de quelques Chinois hautement qualifiés. La République de Chine étant partie prenante de la Société des Nations, elle avait envoyé des représentants à Genève. Après l'échec de celle-ci, beaucoup de ses organes, par exemple l'Organisation internationale du travail (OIT), continuèrent à fonctionner pour finalement être rattachés à l'Organisation des Nations Unies (ONU). Les délégués de la Chine se sont ainsi succédés jusqu'au moment où les communistes ont pris le pouvoir en Chine continentale et où certains de ces

17 La migration chinoise vers l'Europe a débuté au XIXe siècle, mais elle s'est surtout accentuée lors de la Première Guerre mondiale, période durant laquelle un certain nombre de Chinois provenant de Wenzhou et de la ville proche de Qingtian (Sud du Zhejiang) ont été embauchés pour creuser des tranchées ou comme main-d'œuvre de l'industrie d'armement française. Une fois la guerre finie, nombre d'entre eux sont restés et on a assisté à la formation de petites communautés chinoises dans les grands centres urbains. Ces premiers noyaux ont été des points de référence pour les flux migratoires suivants en provenance du Zhejiang ou du Fujian (Campani et al., 1994), majoritaires en Italie, en Hollande ou en France. 
fonctionnaires décidèrent de rester en Suisse et de faire venir leur famille. Cette démarche fut facilitée par le fait que si la Suisse a reconnu la République populaire de Chine dès janvier $1950^{18}$, l'ONU a continué de considérer la République de Chine, comme seule représentante officielle de la Chine jusqu'en 1971. Les récits de quelques interlocuteurs montrent que les Chinois se comptaient sur les doigts d'une main à l'époque ${ }^{19}$.

De fait, dans les années 1950-1960, ce ne sont que quelques familles riches et éduquées, qui jouissaient de très bonnes situations, tant économique que politique, sous le gouvernement de Tchang Kaï-Chek, désormais replié sur l'île de Taïwan, qui s'installèrent en Suisse. Beaucoup sont arrivés en Suisse pour fuir le régime communiste, après avoir résidé d'abord à Hong-Kong, voire Taïwan, quand les membres de leur famille étaient dispersés dans le monde. Ce faisant, ils voulaient préserver leur situation financière dans un pays ayant la réputation d'être paisible. Ainsi, les uns étaient les enfants de fonctionnaires internationaux, les autres étaient des intellectuels. On notera d'ailleurs que les femmes avaient pour la plupart mené des études universitaires poussées.

Après la reconnaissance officielle par l'ONU de la République populaire de Chine en 1971, les fonctionnaires des organisations internationales ont été remplacés, petit à petit, par des ressortissants de Chine populaire. Ainsi, les diplomates, interprètes et traducteurs de ces organisations ne se composent pas de personnes de même origine régionale, ni politique. Les ressortissants de Chine populaire avaient un statut particulier pour des raisons propres à leur gouvernement, qui ne leur permettait de rester que de façon temporaire en Suisse. Ils devaient reverser une partie de leur traitement à leur pays et vivaient chichement, en célibataires. Un changement de statut, dans les années 1990, leur a permis de rester et de faire venir leur famille, et c'est ainsi que s'est constitué un petit groupe de personnes, résidant en Suisse depuis quinze à vingt ans, voire trente ans, qui vit dans un cercle très international, quoique relativement fermé. La plupart d'entre eux ne parlent pas le français et évoluent dans des réseaux sinophones ou anglophones.

Après une longue période de fermeture à l'égard des pays de l'Ouest, les échanges académiques ont repris au début des années 1980, moment où les premiers étudiants boursiers ont été envoyés par la Chine populaire. On trouve également, dès la fin des années 1980, des étudiants qui financent leurs études. ils étaient membres de familles d'intellectuels persécutés pendant la révolution culturelle et ont profité de la politique de réforme et d'ouverture pour fuir leur pays. À l'époque, la Suisse représentait, à leurs yeux, l'occasion d'effectuer les études qu'ils n'avaient pas pu faire en Chine. Pour autant, la plupart d'entre eux n'ont pas pu rester, même s'ils y ont séjourné durant de nombreuses années et ont mené à bien des études très poussées ${ }^{20}$. Ceux qui sont toujours en Suisse

18 Pour plus d'information sur l'histoire diplomatique entre la Suisse et la Chine, voir Coduri, 2004 ; Coduri et Keller, 2004 ; Baumberger et Keller, 2004.

19 M. C., Genève, 2006 ; Mme L., Nyon, 2007 ; M. Q., Genève, 2007.

$20 \mathrm{Il}$ apparaît qu'il est très difficile pour ceux qui ont séjourné sur le territoire de rester après des études, ce que déplorent beaucoup des personnes concernées que nous avons interrogées, tout comme certains membres de l'administration qui dénoncent le fait que la Suisse forme (et donc paie pour) des personnes qui iront travailler ailleurs et ne peut donc pas bénéficier de leurs savoirfaire. 
racontent toutefois avoir été très solidaires des autres étudiants boursiers, car ils étaient fort peu nombreux et avaient très peu de ressources économiques. On relève d'ailleurs une forte homogamie, puisque les personnes rencontrées ont toutes épousé un frère ou une sœur d'un autre étudiant chinois.

Originaires de différentes parties de la Chine, pour certaines à la retraite, toutes ces catégories de personnes ont fait carrière et sont aujourd'hui relativement aisées. Par ailleurs, qu'elles se soient mariées avec une personne d'origine chinoise ou avec une personne « étrangère » (waiguoren), leurs enfants possèdent dans la majorité des cas la nationalité suisse et se sont mariés, pour les plus âgés, avec des Suisses. Leurs propres enfants, quand ils en ont, ne parlent pas le chinois.

Apatrides ou de nationalité suisse, ces Chinois de l'ancienne génération se définissent eux-mêmes comme un pont entre les deux sociétés, parlant parfaitement le français ou l'allemand et l'anglais, pour ceux qui ont travaillé dans des organisations internationales. Ils ne se reconnaissent pas dans la «nouvelle génération » de Chinois, qu'ils considèrent comme un peu «rustres » et «qui n'ont pas les mêmes valeurs $»^{21}$. Ils regrettent d'ailleurs l'ancien temps, lorsque les Chinois étaient considérés comme faisant partie d'une élite, c'est-à-dire, soit des étudiants choisis par le biais d'examens extrêmement sélectifs par leur gouvernement, soit des fonctionnaires internationaux. Comme l'explique un interlocuteur, " avant on nous demandait : "votre père est diplomate?", maintenant quand on voit un Chinois, on lui demande, "votre père est restaurateur?" ${ }^{22}$.

Contrairement à d'autres pays d'Europe, les premiers Chinois de Suisse sont arrivés dans des cadres successifs très précis et étaient fort peu nombreux. Ils ne provenaient pas d'une région particulière et étaient des personnes d'un niveau socioculturel et économique élevé (hormis pour les étudiants boursiers qui vivaient très chichement) (Xu, 1956 ; Yao, 1988). Ils ne se sont pas lancés dans les activités commerciales qui, ailleurs en Europe, ont servi de base à un flux migratoire croissant. Voilà qui explique en partie pourquoi, à l'époque, en parallèle des politiques d'immigration restrictives mises en place dès les années 1960 (Piguet, 2004), la Suisse n'a accueilli qu'un petit nombre de Chinois.

\section{Le contingent des Chinois d'Asie du Sud-Est}

La situation politique en Chine continentale après 1949 explique également le faible flux. Hormis des Hongkongais, peu de personnes ont immigré pendant la période de la Guerre froide, car les politiques en matière d'émigration étaient très restrictives aussi bien en Chine populaire qu'à Taïwan. La politique suisse à l'égard des réfugiés ${ }^{23}$ a permis l'arrivée en 1963 de 1000 Tibétains fuyant la domination chinoise, puis entre 1978 et 1981, d'un contingent de 5000 personnes sous l'égide du Haut-commissariat pour les

21 Mme L., Nyon, 2007 ; M. Q., Genève, 2007 ; Mme W., Berne, 2007 ; M. D., Lausanne, 2007.

22 M. C., Genève, 2008.

23 La Suisse compte la deuxième plus grande communauté tibétaine hors d'Asie. 
réfugiés ${ }^{24}$. Appartenant pour la plupart à des minorités chinoises, elles provenaient de pays du Sud-Est asiatique où elles subissaient diverses formes de persécutions. Elles se sont installées en nombre dans la région de Lucerne et dans quelques autres villes de Suisse, où elles se sont lancées dans la restauration ${ }^{25}$. Celle-ci reste aujourd'hui encore, leur domaine d'activité privilégié.

L'arrivée de ces huaqiao, comme les autres Chinois de Suisse appellent ces Chinois du Vietnam, du Laos ou du Cambodge ${ }^{26}$, a constitué un premier bouleversement. De fait, l'arrivée de 5000 personnes n'est pas anodine, quand on sait qu'il n'y avait pas plus de 300 Chinois résidant en Suisse à l'époque ${ }^{27}$ et ce, d'autant plus, que s'ils se sentent « culturellement » et « ethniquement » Chinois, leur langue maternelle n'est généralement pas le mandarin ${ }^{28}$. Ce sont ces Chinois d'Asie du Sud-Est qui les premiers ont créé des associations de Chinois en Suisse (comme on le verra dans la troisième partie) et qui, jusque dans les années 2000, ont organisé les activités autour des principales célébrations chinoises (notamment le Nouvel An). Néanmoins, ces personnes sont souvent montrées du doigt par nos interlocuteurs hautement qualifiés (qu'ils viennent de Chine populaire ou de Taïwan), qui les présentent comme n'ayant pas ou peu d'éducation et comme vivant en relative autarcie ${ }^{29}$. Or, les Chinois d'Asie du Sud-Est qui ont pu venir en Europe sont généralement issus des familles aisées - celles qui avaient suffisamment de ressources pour payer les frais importants pour embarquer sur les bateaux des boat-people. C'est davantage leur position sociale en Suisse qui les distingue de nos autres interlocuteurs, dont les occupations sont plus valorisées.

\section{Les « nouveaux migrants » : expatriés et étudiants}

Les migrations en provenance de la Chine populaire n'ont repris qu'après le lancement des politiques de réformes en place à la fin des années 1970. Elles ont pris de

24 Il n'en va pas de même pour les ressortissants de la République de Chine populaire. Ainsi, aucun des dissidents arrivés en Suisse après les événements de 1989 n'a obtenu l'asile politique. La lenteur des procédures administratives les a en effet incités à tenter leur chance dans d'autres pays d'Europe. D'autres témoignages d'étudiants résidants en Suisse à l'époque laissent penser que le gouvernement suisse a eu une attitude bienveillante envers leurs demandes de prolongation de visa.

25 En 1970, il n'y avait que deux restaurants chinois en Suisse (M. C., Genève, 2006).

26 L'usage par les Chinois de Suisse de ce terme est intéressant puisqu'il concerne généralement tous les Chinois et leur descendance vivant à l'étranger (voir la note 5). Ici, le terme est restreint aux Chinois provenant d'Asie du Sud-Est, en opposition à ceux qui sont nés en Chine continentale ou à Taiwan. Quand a priori ce terme devrait faire référence à ceux qui ont encore la citoyenneté chinoise, son usage marque ici une distinction de catégorie sociale, ainsi qu'une distinction entre catégories de migrants chinois.

27 Officiellement, il y avait 212 Chinois résidant en 1978 (ODM).

28 Les Chinois d'Asie du Sud-Est parlent notamment le Cantonnais ou le Hakka.

29 «Si vous voulez étudier la "communauté" chinoise, allez voir les huaqiao », nous a expliqué une interlocutrice (Mme L., Berne, 2007). 
l'ampleur dès les années $1990^{30}$. En Suisse pourtant, la politique dite « des trois cercles », en vigueur de 1991 à 1998, en a très largement freiné l'essor, tout comme la politique des deux cercles qui lui a succédé ${ }^{31}$. Celle-ci se base sur le niveau de qualification des personnes désirant immigrer, si bien que tous les ressortissants des pays non membres de l'Union européenne ${ }^{32}$ ne peuvent immigrer que sous condition. Dans les faits, cette politique restreint l'immigration en Suisse à la seule main-d'œuvre hautement qualifiée.

Hormis les personnes qui occupent des fonctions particulières dans les organisations internationales, les Chinois en général, en tant que ressortissants de pays non européens, dépendent de la politique suisse d'immigration et ne peuvent s'installer dans le pays que dans le cadre d'un contingent annuel d'autorisation de séjour pour les extracommunautaires, réparti selon les cantons. En conséquence, la plupart des Chinois entrés en Suisse après 1990 présentent des caractéristiques socio-économiques relativement proches de celles des fonctionnaires internationaux. Il s'agit de personnes qualifiées, ayant effectué des études de troisième cycle en Chine ou à l'étranger et qui ont été embauchées par des grands groupes industriels ou financiers. Ils y occupent des postes à responsabilité et ont des revenus élevés. Leurs trajectoires s'inscrivent parfaitement dans la dynamique des mobilités internationales observées pour d'autres nationalités (Nedelcu, 2004).

La politique chinoise encourage, dès les années 1990, les projets d'études à l'étranger (Zhang, 2003). Et c'est la principale raison pour laquelle le nombre de résidents chinois en Suisse augmente de manière importante dès les années 1990 (Figure 1), et ce, d'autant plus que la Suisse est réputée pour ses écoles privées qui voient d'un bon œil l'arrivée des étudiants chinois. Cette catégorie est cependant loin d'être homogène (Xiang, 2003).

30 Les changements économiques et sociaux intervenus en Chine populaire, tout comme la loi de 1985 autorisant ses ressortissants à avoir un passeport dans le cas où ils auraient des lettres d'invitation de l'étranger, ont eu une incidence importante sur le phénomène migratoire chinois vers l'Europe, qui n'était pas une destination traditionnelle et où on constate effectivement une augmentation de la population chinoise liée à la recrudescence d'étudiants et de travailleurs qualifiés.

$31 \mathrm{La}$ politique des trois cercles distinguait les étrangers selon leur provenance. Le droit de libre circulation n'était accordé qu'aux seuls membres de l'Association européenne de libre échange (AELE), avec une extension à certains pays européens ou non européens, comme les États-Unis et le Canada (le deuxième cercle). En revanche, les ressortissants des pays dits du " cercle extérieur » n'avaient, en principe, aucune possibilité de se voir admis. Étienne Piguet relève le caractère extrêmement discriminatoire de la distinction entre deuxième cercle (médian) et troisième cercle (extérieur) puisqu'elle est « basée sur une série de critères que doivent cumulativement respecter les pays du cercle médian : respect des droits de l'homme, appartenance "à un milieu culturel présentant des conditions de vie proches des nôtres (caractère européen au sens large)", relations commerciales solides avec la Suisse et tradition de bonnes relations de recrutement de maind'œuvre » (2004 : 59). Cette politique a fait l'objet de nombreuses critiques, notamment en raison de ses conséquences sur la possibilité de recruter dans le milieu de l'hôtellerie et du bâtiment (ibid. : 59-63).

32 Un accord de libre circulation est effectif depuis 2002. 
Beaucoup de personnes sont arrivées dans le cadre de coopération scientifique officielle ${ }^{33}$. Ainsi des étudiants boursiers qui sont le plus souvent de jeunes hommes inscrits en doctorat ou en post-doc et qui travaillent dans l'une des deux écoles polytechniques fédérales (à Zurich et à Lausanne). Ces personnes pensent avant tout à parfaire leurs compétences afin de devenir cadres dans des entreprises multinationales. Beaucoup disent avoir choisi la Suisse pour la réputation de ses écoles et avoir comparé les montants des bourses entre plusieurs pays avant de faire leur choix. Très mobiles, leur séjour en Suisse n'est à l'évidence qu'une étape dans leur projet professionnel. Leurs liens avec les membres de la société suisse sont souvent très superficiels, alors que leur niveau socioculturel leur permet d'évoluer dans des cercles internationaux. Ils maîtrisent rarement le français ou l'allemand et utilisent l'anglais comme langue de communication. Disposant de peu de temps libre, ils côtoient le plus souvent d'autres collègues étrangers dans leur laboratoire ou des compatriotes.

Le profil des étudiants qui financent leurs études est très différent de celui des étudiants boursiers. Ils arrivent par leurs propres moyens et souvent n'ont pas pu être admis dans les universités chinoises de renom - le système éducatif chinois étant très sélectif (Sztanke, 2005). Leurs parents ont généralement dépensé une grande partie de leurs économies pour les aider à poursuivre leur formation à l'étranger, dans une université ou dans une école privée (souvent des filières touristiques ou des écoles hôtelières). Pour ces personnes, la durée des études peut être longue. Elles doivent souvent commencer par des cours de langue, voire parfois reprendre leurs études, car elles n'ont pas pu obtenir d'équivalence. Du fait de leurs faibles moyens, la plupart de ces étudiants travaillent et font différents petits métiers dans des établissements où leurs capacités langagières sont valorisées (restaurants chinois ou centres de médecine chinoise) ou dans des entreprises suisses. Certains (le plus souvent des femmes) se sont mariés avec des Suisses et se sont installés définitivement.

Depuis 2001, le gouvernement suisse cherche à sélectionner les étudiants, afin de n'accueillir que les meilleurs et d'éviter que les visas d'étudiants ne deviennent un « alibi » pour rentrer sur le territoire suisse ${ }^{34}$. Il est donc demandé aux étudiants non ressortissants des pays de l'Union européenne, de l'AELE, et des grands pays anglo-saxons, de

33 Il existe de nombreux échanges scientifiques entre les deux pays. Certains chercheurs invités effectuent des recherches dans le cadre d'un institut partenaire suisse durant une année puis reprennent leur poste en Chine. S'ils côtoient les étudiants, leurs trajectoires migratoires s'en différencient, car à l'inverse des étudiants, leur parcours se limite à un seul pays, la Suisse, et pour une courte durée.

34 Le gouvernement suisse craint en effet une forme organisée de migration illégale via les écoles privées, suite à la soudaine « disparition », en novembre 2005, d'environ soixante-dix étudiants, originaires de la province du Zhejiang, qui n'ont pas suivi les études envisagées et sont partis sans que les autorités suisses ne sachent où. Voir http://www.sinoptic.ch/politique/ecoles/. 
s'engager par écrit à quitter la Suisse après leurs études ${ }^{35}$. Depuis décembre 2005, cette politique s'accentue puisqu'une directive concerne notamment les demandes de visa pour étudiants en provenance de République populaire de Chine ${ }^{36}$. Elle stipule que celles-ci seront en principe refusées ${ }^{37}$.

Mais les politiques migratoires ne suffisant pas à fermer les frontières, il existe également des personnes sans-papiers. Elles sont peu nombreuses et inégalement réparties sur le territoire. Les hommes sont majoritaires, pour la plupart originaires du Fujian ${ }^{38}$, mais nous avons également rencontré des personnes originaires de grandes villes comme Shanghai ou Pékin et qui se retrouvent en situation d'autant plus précaire qu'elles ne peuvent compter sur les solidarités régionales. Certaines sont arrivées avec des visas étudiants ou comme simples touristes, d'autres clandestinement. Leur motivation migratoire est avant tout économique, et dans bien des cas, ces personnes n'envisagent de séjourner en Suisse que temporairement. Tous leurs efforts sont tournés vers le travail et la possibilité de gagner de l'argent afin de faire vivre leur famille et de rentrer en Chine au plus vite. Peu d'entre elles maîtrisent vraiment le français, et elles vivent largement en marge de la société locale. Si, comme beaucoup de migrants sans-papiers chinois ailleurs en Europe, ces personnes travaillent surtout dans la restauration, dans le cas suisse, elles ne sont pas exclusivement employées par des patrons chinois. Beaucoup travaillent pour des employeurs locaux propriétaires de restaurants suisses, italiens ou encore thaïlandais, où les rémunérations sont très faibles.

Pour résumer, depuis les années 1990, ce sont avant tout des Chinois qualifiés de Chine populaire qui sont arrivés en Suisse, qu'ils soient cadres ou étudiants. Ainsi, les nouvelles tendances des migrations chinoises en Suisse reflètent les profonds changements sociaux survenus en Chine : avec d'une part, l'élévation du niveau d'éducation, qui est à l'origine de la migration de professionnels hautement qualifiés, et d'autre part

35 En la matière, les Chinois sont traités comme tous les ressortissants du « deuxième cercle ». Mais le gouvernement suisse leur porte une grande attention. En 1996 en effet, une circulaire sur l'inscription des étudiants étrangers dans les écoles privées suisses concernait la Chine en particulier (avec cinq autres pays : Circulaire du 2 février et du 31 juillet 1996 intitulée « Inscription d'étudiants étrangers dans des écoles privées suisses/immigration illégale en provenance du Pakistan, d'Inde, du Bangladesh, de Sri Lanka, d'Afghanistan et de Chine »). Cette circulaire a été remplacée en 2001, par une seconde circulaire générale pour les non-ressortissants des pays des premier et deuxième cercles.

36 Et de douze autres pays : l'Algérie, le Bangladesh, le Burundi, le Cameroun, la Guinée, Haïti, l'Irak, la Libye, le Myanmar, la République démocratique du Congo, la République du Congo, le Rwanda et le Soudan.

37 Qui plus est, les cantons qui, en principe, ont compétence en matière de visa d'études doivent en référer désormais à l'administration fédérale. Cela dit, cette directive ne semble pas avoir d'impact pour les étudiants qualifiés qui viennent dans le cadre d'échanges officiels : http://www. bfm.admin.ch/etc/medialib/data/migration/rechtsgrundlagen/weisungen_und_kreisschreiben/ weitere_weisungen.Par.0035.File.dat/F_studenten_china_281205_f.pdf (consulté le 9 février 2009).

38 Il s'agit d'une région migratoire très connue. Les gens du Fujian forment le gros des effectifs des derniers migrants chinois aux États-Unis et en Grande-Bretagne. Sur la diaspora du Fujian, voir notamment Frank Pieke et al. (2004). 
l'apparition d'une nouvelle " classe moyenne » qui peut désormais voyager et envoyer ses enfants à l'étranger (Pieke, 2007). Aujourd'hui, comme pour d'autres pays, le statut d'étudiant représente la voie d'entrée en Suisse la plus courante. On remarquera d'ailleurs que les deux tiers des travailleurs étrangers qualifiés dans les pays de l'OCDE ont été préalablement étudiants (Meyer et Hernandez, 2004). Ces personnes ont des pratiques proches de celles des expatriés - c'est-à-dire des employés d'entreprises multinationales ou des scientifiques qui résident à l'étranger, sur une base volontaire et temporaire (Cohen, 1977), et qui choisissent leur destination en fonction d'intérêts professionnels et économiques ${ }^{39}$.

\section{D'une migration d'élite à l'autre}

Cette présentation des migrations chinoises en Suisse selon les époques souligne l'influence des contextes politiques, suisse et chinois, sur les modalités d'arrivée et le type de population concernée. Elle permet également de souligner la spécificité du cas suisse en regard des autres pays européens. Les migrations chinoises ont toujours été faibles et majoritairement composées de cols-blancs, ce qui a eu des conséquences sur le type de personnes venues s'installer par la suite. Ce qui explique aussi en grande partie le faible nombre des sans-papiers chinois en Suisse, puisqu'il n'y a jamais eu de véritable marché du travail ethnique. En effet, plus que les mesures politiques face à l'immigration, c'est le marché du travail qui a joué sur le taux de présence de personnes en situation irrégulière (Longchamp et al. 2005).

Cette typologie permet également de souligner un bouleversement important dans la façon dont cette population se structure avec l'arrivée des Chinois de Chine populaire. On a, en quelque sorte, à faire à un changement d'élite. Il importe donc de comprendre comment cette population s'organise aujourd'hui et ce, d'autant plus, qu'on voit se dessiner de nouvelles formes d'allégeance.

\section{DE NOUVELLES FORMES D’ORGANISATION}

L'accroissement de la population chinoise en Suisse s'est accompagné d'une recrudescence de nouvelles associations, puisqu'on a observé la création d'au moins sept d'entre elles depuis 2003 - pour une population, on l'a vue qui reste relativement réduite. Le 27 septembre 2009, près de Berne, elles se sont regroupées pour célébrer le $60^{\mathrm{e}}$ anniversaire de la République populaire de Chine. Cette manifestation a réuni plus de 550 personnes ${ }^{40}$ - une première en Suisse - et marque une sorte de rupture dans les formes d'organisations préalables.

39 Généralement le terme expatrié est utilisé pour parler des personnes des pays « développés » qui travaillent dans les pays moins favorisés, mais il s'applique très bien à la population hautement qualifiée de Chinois résidants en Suisse.

$40 \mathrm{http} / /$ www.swissinfo.ch/chi/swissinfo.html?siteSect=105\&sid=11277097. 
En Suisse, comme ailleurs, la façon dont les communautés d'origine étrangère se sont donné des formes associatives a varié dans le temps. Le cas des Chinois ne déroge pas à cette règle et on peut distinguer trois périodes qui correspondent aux années 1980, 1990 et 2000.

\section{Les années 1980 : des associations d'entraide et d'amitié}

Avant les années 1980, en raison du faible nombre de Chinois en Suisse et en raison des modalités de leur venue, il n'y avait pas d'associations de Chinois, pas même celles que l'on retrouve typiquement chez les Chinois d'Outre-mer, c'est-à-dire des regroupements en fonction de l'origine régionale ou patronymique. Ce n'est qu'après l'arrivée du contingent de Chinois d'Asie du Sud-Est, à la fin des années 1970, qu'on a vu se créer des associations d'entraide. Les informations récoltées par Yao (1988), un membre du bureau qui faisait office de représentation diplomatique de la République de Chine ${ }^{41}$, montrent qu'il y a vingt ans, les organisations de Chinois de Suisse regroupaient avant tout des Chinois d'Asie du Sud-Est ${ }^{42}$. Il en existait trois à la fin des années 1980, toutes en Suisse allemande ${ }^{43}$, région où ces personnes se sont installées en majorité. Les objectifs de ces associations étaient semblables : renforcer la cohésion de la communauté et créer des liens entre les Chinois d'Asie du Sud-Est, les plus nombreux à l'époque. Des trois, seule la Fédération des Chinois de Suisse est encore active aujourd'hui, bien que sur le déclin. Elle a compté jusqu'à 3000 membres et elle est encore en mesure de les mobiliser, notamment lors des grands événements, par exemple une soirée de bienfaisance pour récolter des fonds en faveur des victimes du tremblement de terre du Sichuan, début 2009 ${ }^{44}$. Durant de nombreuses années, cette association a organisé toutes les célébrations du Nouvel An chinois. Avec le soutien logistique de la République de Chine, elle a longtemps organisé des cours de langues et des camps d'été pour les enfants. À l'époque, ces trois associations soutenaient le gouvernement nationaliste.

D'autres associations existaient également ${ }^{45}$, mais de façon plus informelle. L'Amicale des Chinois de Genève (rineiwa ruishi huaren lianyihui), par exemple, a été créée en 1983. Elle n'a jamais eu de statuts à proprement parler et ses membres ne

$41 \mathrm{La}$ Suisse ayant reconnu la République populaire de Chine en 1950, elle n'a officiellement pas de relations diplomatiques avec la République de Chine. Celle-ci a tout de même deux bureaux de représentation à Berne et à Genève, appelés Délégation culturelle et économique de Taipei.

42 Bien qu'on puisse également considérer qu'une association de Chinois d'Asie du Sud-Est est une association « régionale », elle se distingue néanmoins des associations régionales classiques (tongxianghui) qui font davantage référence à une province de Chine continentale (le Guangdong ou le Fujian par exemple).

43 Il s'agit respectivement de l'Association d'amitié des Chinois de Suisse (ruishi huaren lianyihui), créée à Lucerne en 1980; de l'Association suisse d'entraide et d'amitié (ruishi lianyou huzhuhui) créée à Bâle en 1981; de la Fédération des Chinois de Suisse (ruishi huaren lianhehui; Der Bund der Chinesen in der Schweiz), créée à Lucerne également, en 1985.

$44 \mathrm{http}: / /$ sites.google.com/site/charityevent2009/supporters.

45 Ces associations ne sont pas mentionnées par Yao (1988), représentant du gouvernement de la République de Chine et qui n'a recensé que les associations proches de son gouvernement. Elles ont été cependant mentionnées par certains de nos interlocuteurs. 
payaient pas de cotisation. Lieu de rencontre, elle regroupait avant tout des interprètes et des traducteurs travaillant dans les organisations internationales, généralement non francophones, à qui elle servait également de réseau d'entraide. Les hommes se retrouvaient pour pratiquer le taiqi, tandis que pour les femmes, l'activité principale était la transmission du chinois aux enfants (aujourd'hui, tous adolescents ou adultes). Ces femmes ont été à l'origine d'une des deux écoles de langue chinoise de Genève. En parallèle, les étudiants, peu nombreux à l'époque et sans grande ressource, expliquent qu'il y avait de forts réseaux de pairs et qu'ils se soutenaient mutuellement. Ceux qui étaient venus étudier à Lausanne ou à Zurich (dans les écoles polytechniques) expliquent qu'ils s'étaient organisés pour préparer des repas en commun et pour donner les informations nécessaires aux nouveaux venus ${ }^{46}$.

\section{Les années 1990 : le processus d'affirmation d'une « identité chinoise »}

Le début des années 1990 est une période charnière : en 1991, deux associations d'assise purement régionale virent le jour - l'Association suisse des gens de Chaozhou (ruishi chaozhou tongxianghui) et l'Association suisse des Cantonais (ruishi guangdong tongxianghui). Elles appartenaient également à un réseau international d'associations similaires $^{47}$. Outre l'entraide et le soutien objectif qu'elles partageaient avec les associations créées dans les années 1980, elles visaient également à préserver et diffuser « la » culture chinoise.

L'apparition sur la scène suisse de ces deux associations ne relève pas entièrement du hasard. Elle est liée à la politique de réformes et d'ouverture engagées en Chine à la fin des années 1970, et à l'évolution concomitante, sur place, du point de vue sur les émigrés, considérés dès lors comme partie intégrante de la "modernisation socialiste " du pays (Guérassimoff, 2006 ; Nyiri, 2004 ; Thuno, 2001 ; Louie, 2000). Ainsi, une des stratégies du gouvernement de Pékin a consisté à soutenir les associations de Chinois d'Outre-mer afin d'établir des contacts avec leurs membres (Hong, 1998 ; Barabantseva, $2005)^{48}$. Pour Mette Thuno, qui a analysé des archives administratives des provinces du Fujian et du Guangdong, l'aspect frappant de cette évolution est la « racialisation » des discours. "Les Chinois d'Outre-mer devaient être considérés comme intimement liés à la Chine, de par leur race, leur lieu de naissance et leur culture » (2001 : 921) ${ }^{49}$. S'attirer les faveurs des Chinois d'Outre-mer, généralement proches du gouvernement au pouvoir en République de Chine représentait non seulement un moyen d'attirer des fonds vers la Chine continentale, mais permettait également de "promouvoir le développement de

46 M. D, Lausanne, 2007 ; M. T, Genève, 2007.

47 Mme C., Bienne, 2008. Sur la question des réseaux d'associations chinoises, voir notamment Li, 1998 et Hong, 1998.

48 On parle d'une stratégie pro-diaspora, de « wei guo fuwu », ce qu'on traduit généralement par « servir le pays depuis l'étranger».

49 Un article connu de Fitzgerald (1970) traite, lui, des politiques à l'égard des Chinois d'Outre-mer avant les réformes. Il était alors vivement conseillé aux Chinois d'Outre-mer de se fondre dans le pays dans lequel ils vivaient. La République de Chine entretenait, elle, des liens très étroits avec les communautés chinoises en Asie du Sud-Est. 
contacts virtuels à travers le détroit (de Tä̈wan) », et ainsi de favoriser l'« unification avec Tä̈wan » (2001: 921-922) ${ }^{50}$. Et de fait, les deux associations régionales ont participé à un meeting en 2004, qui affirmait leur opposition à l'indépendance de Taïwan ${ }^{51}$. Leur création, en 1991, relève en partie d'une stratégie du gouvernement de Chine populaire pour s'assurer l'allégeance des Chinois vivant en Suisse, qui à l'époque était davantage proche du Guomindang (Yao, 1988). Cette affirmation se voit confirmée par la personnalité des divers présidents, dont les liens avec le gouvernement de Chine populaire sont, pour certains, évidents - ils participent à la plupart des manifestations organisées par l'ambassade de la République populaire de Chine à Berne.

La tension entre les « deux Chines » et la compétition des deux gouvernements semblent donc expliquer la création de certaines associations dans les années 1990. Comme l'affirme un interlocuteur, "chaque fois que les uns créent quelque chose, les autres doivent le faire aussi ». Il critique d'ailleurs la façon dont ces associations sont souvent créées "par le haut». " [Les représentants diplomatiques] ont reçu des instructions de leur gouvernement et ils les appliquent». En parlant de toutes ces associations, ce même interlocuteur insiste : "Ils sont invités à Tä̈wan ou en Chine pour un congrès ou autre, mais ils ne représentent rien! ${ }^{52}$.

Cette situation explique sans doute le peu de visibilité de ces associations parmi nos interlocuteurs. En effet, rares sont les personnes interrogées qui nous en ont parlé53. Toutefois, s’il existe de véritables intérêts politiques à développer des associations favorables au gouvernement de Pékin ou de Taipei, certaines d'entre elles sont investies par leurs membres.

Les écoles peuvent être considérées comme faisant partie de ces nouveaux organismes étatiques visant à maintenir les liens avec les Chinois vivant à l'étranger et avec les personnes d'ascendance chinoise - et ainsi créer un sentiment d'unité. Mette Thuno (2001) et Elena Barabantseva (2005) insistent en effet sur la promotion, depuis les années 1990, de l'éducation et de la culture chinoise comme un des instruments de cette politique - le gouvernement de Pékin reprenant à son compte une politique que le gouvernement nationaliste avait développée de longue date en Asie du Sud-Est. Ainsi, durant cette décade, la question de la transmission de la langue aux deuxièmes générations, et éventuellement aux Occidentaux, a constitué un des thèmes les plus mobilisateurs. La plupart des associations créées en Suisse, au cours de ces années, sont organisées autour d'écoles du week-end pour les enfants. Leur nombre est d'ailleurs aujourd'hui étonnamment important en regard

50 Pour plus d'informations sur les diverses politiques de la Chine populaire à ce sujet, voir : Barabantseva, 2005 ; Guérassimoff, 2006 ; Hong, 2005 ; Nyiri, 2004 ; Thuno, 2001.

51 http://www.cns.hk:89/news/2004/2004-10-08/26/491440.shtml.

52 M. C., Genève, 2009.

53 Lors de la célébration du 60e anniversaire de la République populaire de Chine, ma voisine de table, une ancienne étudiante mariée à un Suisse, m'expliquait qu'elle avait appris de cette commémoration totalement par hasard. Elle a passé le reste de l'après-midi à demander autour d'elle s'il existait des associations de Chinois en Suisse, pays qu'elle habite depuis une dizaine d'années. 
de la taille de la communauté. Nous en avons dénombré pas moins de seize ${ }^{54}$, dont quatre bénéficient du soutien de la République de Chine.

La question de transmettre « la » culture et « la » langue chinoise (ici le mandarin), permet de regrouper des personnes de tous horizons. Les femmes jouent un rôle central dans cette dynamique, avec le soutien des représentations diplomatiques. À Zurich, diverses associations offrent des cours de langue pour les enfants et les adultes, et un jardin d'enfants. À Genève et à Lausanne, il existe trois écoles chinoises où l'on enseigne le mandarin. À Lausanne, l'école a été créée à l'initiative des femmes d'étudiants « de la première génération » dans le but d'apprendre à leurs enfants à lire et à écrire en chinois. Elle a ensuite accueilli un plus large public, avant de perdre petit à petit ses effectifs. Elle serait aujourd'hui sur le déclin, faute de bénévoles. À Genève, deux écoles cohabitent. La première, déjà évoquée plus haut, a été créée en 1991 à l'initiative des femmes des interprètes et traducteurs des organisations internationales. Elle est en partie soutenue par le gouvernement de Chine populaire et utilise des manuels en caractères simplifiés ${ }^{55}$. La seconde, désignée comme l'école « taïwanaise », a vu le jour plus récemment, sous l'impulsion de la représentation économique de Taipei et grâce à la volonté de mères taïwanaises. Les cours sont cette fois dispensés en caractères non simplifiés.

Selon nos observations, ces deux écoles genevoises attirent toutes deux des publics très variés : enfants de migrants chinois ou taïwanais, de couples mixtes, de Chinois d'Asie du Sud-Est, ainsi que de personnes ayant un intérêt pour la Chine. Il faut noter que parmi les personnes qui fréquentent ces deux écoles, la question de l'écriture enseignée (en caractères simplifiés ou non) ne conduit pas au clivage entre ressortissants de Chine populaire d'une part et de Taïwan d'autre part. Les parents choisissent davantage l'école en fonction de critères pratiques, tels que les horaires ou la proximité. Il n'en reste pas moins qu'au-delà du désir compréhensible pour des parents de transmettre leur langue maternelle à leurs enfants se trouvent également des politiques gouvernementales plus complexes (Lieber, 2008). Pour preuve, les diverses écoles de Chinois de Suisse cherchent à se regrouper en réseau. À l'initiative des chargés de l'éducation des représentations diplomatiques de chacune des « deux Chines », il existe désormais deux réseaux parallèles d'enseignement du chinois en Suisse ${ }^{56}$.

$54 \mathrm{http} / /$ www.swissinfo.ch/chi/search/Result.html?siteSect=882\&ty=st\&sid=1582218. Aux USA, on compte environ 700 écoles de ce type pour une population estimée à 2,7 millions (voir le site Internet de National council of associations of Chinese language schools : http://www.ncacls. org). Genève, Zürich, Lucerne ou Berne en ont deux alors que leur population chinoise ne dépasse pas le millier.

55 Deux systèmes graphiques coexistent : les caractères simplifiés par le gouvernement communiste adoptés en 1958 et l'écriture traditionnelle (non simplifiée) qui reste en usage à Taiwan, Singapour et encore largement à Hong-Kong. L'utilisation de caractères simplifiés ou non est donc un marqueur d'une allégeance à la Chine populaire ou au gouvernement de Taiwan.

56 L'année 2007, a vu la création d'une association d'enseignants de chinois soutenue par la République de Chine (la Fédération suisse des professeurs de chinois, ruishi zhongwen jiaoshi lianhehui) et une autre par la République populaire de Chine (l'Association suisse des professeurs de chinois, ruishi zhongwen jiaoshi xiehui). 


\section{Les années 2000 : la défense des intérêts économiques et les « nouveaux migrants »}

Les années 2000 sont marquées par une évolution significative des modalités d'organisations des Chinois de Suisse. Prenons l'exemple de l'association Chinese Union Zurich (sulishi zhongguoren xiehui) créée en 2003 et qui a pour objet la promotion des " échanges économiques, culturels, scientifiques et technologiques entre la Chine et la Suisse $\aleph^{57}$. Elle propose de nombreuses conférences, ainsi que des formations pour mieux connaître les pratiques commerciales en Chine. Pour la plupart, ses membres travaillent dans de grandes multinationales ou dans les écoles polytechniques. Autre exemple, l'Association suisse des universitaires chinois en sciences et technologies (ruishi zhongguo xueren keji xiehui), créée à Berne en 2004, se présente comme une « association reconnue parmi les professionnels chinois vivant en Suisse » ayant pour objectif d' " établir des liens avec les scientifiques et les communautés technologiques en Suisse et en Chine $»^{58} .2007$ a vu aussi la création de l'Association des agences de voyages chinoises (huaren lüyouye lianhehui) $^{59}$ et plus récemment, à la fin 2008, la Chambre de commerce chinoise en Suisse a ouvert ses portes à Genève, afin de « promouvoir les relations commerciales entre la Chine et la Suisse $»^{60}$.

Ces exemples témoignent d'une évolution importante. La majorité des associations créées par des Chinois au cours des dix dernières années sont de nature professionnelle et visent à développer les intérêts économiques de leurs membres. Le glissement de petites associations d'entraide des années 1980, qui ciblaient surtout les étudiants boursiers ou les réfugiés, vers des organisations d'une toute autre envergure peut être abordé sous l'angle de l'amélioration du positionnement social des Chinois en Suisse, mais il témoigne surtout des changements qui traversent cette population. Pieke (2007) observe en effet que les regroupements des Chinois, qui caractérisaient les migrations chinoises dites traditionnelles de par le monde, ne constituent plus de nos jours leur unique moyen de subsistance dans un pays étranger, mais consiste davantage en une plateforme, à laquelle les uns et les autres peuvent choisir de participer ou non. Cette analyse vaut également pour la Suisse, puisque les associations d'entraide ont quasi disparu. Toutefois, elle n'est que partielle, car elle évacue toute dimension politique (Nyiri, 1999).

En Suisse, l'augmentation du nombre de « nouveaux migrants », nés en Chine populaire, éduqués dans l'idéologie communiste et ayant gardé un intérêt profond pour l'avenir de leur pays d'origine (Hong, 2005 ; Guérassimoff, 2006), explique l'augmentation et la diversification des organisations. Ces personnes qui ont réussi à l'étranger sont présentées comme des modèles en Chine et de plus en plus d'initiatives gouvernementales visent à développer les liens entre ces Chinois expatriés et la Chine, notamment sur le plan

57 http://www.cuz-online.org/cuz_en/webs/about_cuz/index.php.

$58 \mathrm{http}: / /$ www.sinotech.ch/en/regulations.html.

59 http://www.lotour.com/snapshot/2007-1-8/snapshot_54737.shtml.

$60 \mathrm{http}: /$ www.chinapressusa.com/big5/newscenter/2008-10/19/content_157972.htm; http:/www. swissinfo.ch/chi/news/shortnews/detail.html? siteSect $=106 \&$ sid $=9867340 \& \mathrm{cKey}=12244984780$ $00 \&$ ty $=$ st. 
scientifique et professionnel. Comme le souligne Elena Barabantseva :

« Le gouvernement chinois a fait des efforts considérables pour rassembler des Chinois de toutes les origines et de tous les genres sous la bannière du patriotisme envers la mère patrie. Il semble qu'une intensification de la mobilité, du commerce, des communications, des avancements technologiques et des autres qualités de la globalisation soit bénéfique au programme de modernisation de l'État-nation chinois » $(2005: 23)$.

Ainsi, ces nouveaux migrants sont à l'initiative de la majorité des nouvelles créations, avec le soutien de l'ambassade de la République populaire de Chine. Présentée en général comme un partenaire parmi d'autres, elle occupe en réalité une place centrale. Soulignons que depuis 1998, la Fédération des Chinois de Suisse (ruishi huaren huaqiao lianhe zonghui) affirme représenter tous les Chinois de Suisse et participe à toutes les manifestations officielles de la République populaire de Chine, en Suisse ou à l'étranger. Son président est certes un Chinois d'Asie du Sud-Est, mais il est proche de tous les présidents ou vice-présidents des associations nouvellement créées. Il est à remarquer que ceux-ci forment un petit groupe de personnes qui cumulent les postes à la tête des diverses associations; le président de l'Association des agences de voyages est également vice-président de la Chambre de commerce. Certains de ses amis ont été placés à la tête d'associations plus anciennes, telle que l'Association des gens de Chaozhou, qui est maintenant présidée par un médecin installé à Zurich depuis une quinzaine d'années et qui n'est pas originaire de cette région de Chine. Lorsqu'on lui demande pourquoi il dirige cette association, il explique qu'il a été sollicité parce qu'il a de l'entregent et que personne ne voulait assurer cette fonction : la plupart de ces associations servent davantage de fairevaloir de la politique de Pékin que de représentation effective des Chinois de Suisse. La présence parmi les membres de certaines de ces associations de diplomates de la Chine populaire en offre une autre illustration ${ }^{61}$.

Ces associations viennent concurrencer celles qui ne sont pas dans la proximité de l'ambassade de Chine à Berne. Par exemple, la Communauté chinoise de Genève (rineiwa huaren hui), une des seules associations créées en 2003 qui se présente encore comme une organisation amicale à fonction d'entraide («notamment pour les personnes âgées qui sont très seules ») et qui se veut « apolitique $»^{62}$, organisait chaque année diverses manifestations liées aux grandes fêtes traditionnelles chinoises (Nouvel An, la fête de l'automne, etc.), pour les 1500 Chinois de Genève ${ }^{63}$. Elle invitait aussi bien les représentants de la République populaire de Chine que ceux de la République de Chine. En 2007, toutefois, l'Association suisse des agences de voyages a organisé une fête de Nouvel An parallèle, où les représentants de l'ambassade de la République populaire de Chine avaient une place d'honneur.

61 L'Association suisse des médecins chinois (ruishi huaren zhongyiyao xiehui) a pour membre un secrétaire d'ambassade. Le président de la nouvelle Chambre de commerce chinoise en Suisse est un homme d'affaires, résident de Hong Kong.

62 Mais qui est considérée par certains comme étant « taïwanaise », car créée par un Chinois arrivé en 1950 en Suisse, avec un passeport de République de Chine, avant d'obtenir la nationalité suisse.

63 M. C., Genève, 2006 et 2008. 
Pour résumer, il importe de souligner que les nouvelles formes d'organisation des Chinois de Suisse marquent, comme cela a été analysé ailleurs (Hong, 2005), un resserrement des liens avec la Chine populaire, alors que celle-ci devient une puissance économique et que le nombre de « nouveaux migrants » augmente.

Les nouvelles associations créées montrent le niveau socio-économique de cette population selon les époques, tout en soulignant un changement profond dans les rapports de pouvoir internes. Les associations proches de la République de Chine dans les années 1980 cèdent la place à celles plus proches de la République populaire de Chine. Ce mouvement est visible dès les années 1990.

\section{CONCLUSION}

La population chinoise en Suisse diffère de celles présentes dans d'autres pays d'Europe, alors qu'il n'y a pas eu en Suisse de migrations en chaîne, avec des réseaux de migrants provenant de la même région, comme c'est le cas en France ou en Italie. Au contraire, à l'origine cette population était peu nombreuse et constituée de personnes hautement qualifiées venant de diverses régions de la Chine pré-communiste. Aujourd'hui, elle a été remplacée par des personnes très qualifiées aussi, en provenance de Chine populaire. Elles sont arrivées pour faire des études ou participer à des projets de recherches, ou encore pour travailler dans de grands groupes industriels et financiers. On peut estimer que la population actuelle en Suisse reflète les nouvelles tendances des migrations chinoises dans le monde, puisque l'augmentation de cette population en Europe et aux États-Unis est due principalement à l'arrivée d'étudiants et de cols-blancs en provenance de Chine populaire.

S'il n'existe pas en Suisse de visibilité de la présence de Chinois, que ce soit par l'existence de journaux en mandarin, de Chinatown, de regroupements urbains ou d'associations ayant un véritable poids dans la vie politique, on observe toutefois aujourd'hui une volonté de représenter les intérêts des Chinois de Suisse. En ce sens, le paradoxe mis en évidence pour les États-Unis, qui veut que «l'éviction de barrières structurelles à l'assimilation ne conduit pas à un déclin [...] des organisations ethniques » (Zhou et Kim, 2006 : 249), vaut également pour le contexte suisse. L'augmentation du nombre de Chinois leur permet de s'organiser et de faire valoir les évolutions économiques et politiques de la République populaire de Chine. Ce phénomène permet de saisir les changements qui touchent à l'organisation (même informelle) des Chinois de Suisse et les ruptures qui l'accompagnent. On assiste à une revitalisation des liens avec la Chine populaire, effet de la position économique de celle-ci et de sa politique nationaliste (Hong, 2005). Ces « nouveaux migrants » sont en train de s’imposer parmi les Chinois de Suisse. 


\section{Références bibliographiques}

ANG Ien (1998) Can one say no to Chineseness? Pushing the limits of the diasporic paradigm, Boundary 2, 25 (3), pp. 223-242.

BARABANTSEVA Elena (2005) Trans-nationalising Chineseness: Overseas Chinese policies of the PRC's central government, Asien, 96, pp. 7-28.

BAUMBERGER Eléonore et KELLER Hans (2004) Chine. Les relations bilatérales depuis 1960, in Dictionnaire historique de la Suisse, tome 3, Hauterive, Éditions Gilles Attinger.

BEJA Jean-Philippe (2001) Les Chinois à Paris : une communauté multiple, Migrations et sociétés, 13 (74), pp. 99-106.

BELTRAN Joaquin (2004) La presencia china en el mundo, Barcelona, UOC.

BENTON Gregor and PIEKE Franck (Ed.) (1998) The Chinese in Europe, London, St. Martin's Press.

BROWN Melissa (2004) Is Taïwan Chinese? The impact of Culture, Power and Migration on Changing Identities, Berkley, University of California Press.

CAMPANI Giovanna, CARDECHI Francesco e TASSINARI Alberto (Ed.) (1994) L'immigrazione silenziosa. La comunità cinesi in Italia, Torino, Fondazione Giovanni Agnelli.

CODURI Michèle (2004) La Suisse face à la Chine - une continuité impossible, Thèse de doctorat, Saint-Gall, HSG.

CODURI Michèle et Keller Hans (2004) Chine. Les relations entre la Suisse et la Chine jusqu'en 1960, in Dictionnaire historique de la Suisse, tome 3, Hauterive, Éditions Gilles Attinger.

COHEN Eric (1977) Expatriate communities, Current Sociology, 24 (5), pp. 5-90.

FITZGERALD Stephen (1970) China and the Overseas Chinese: Perceptions and Policies, The China Quarterly, 44, pp. 1-37.

GUERASSIMOFF Carine (2005) République populaire de Chine-Union Européenne : vers un partenariat stratégique en matière migratoire ?, Hommes et migrations : Chinois de France, 1254 , pp. 65-73.

GUERASSIMOFF Carine (2006) Le renouvellement des perspectives transnationales de la Chine, Critiques internationales, 32, pp. 39-52.

HONG Liu (1998), Old linkages, new networks: the globalization of Overseas Chinese voluntary associations and its implications, The China Quarterly, 155, pp. 582-609.

HONG Liu (2005) New migrants and the revival of Overseas Chinese nationalism, Journal of Contemporary China, 43 (14), pp. 291-316.

KENT Robert B. (2003) A diaspora of Chinese Settlement in Latin America and the Carribean, in Laurence J.-C. Ma and Carolyn Cartier Eds., The Chinese diaspora. Space, place, mobility, and identity, Boston, Rowman \& Littlefield.

LACZKO Frank (2003) Europe attracts more migrants from China, Migration Information Source.

LIEBER Marylène (2008) When you look Chinese, you have to speak Chinese. The valorization of a share unified culture and language, Communication, International Forum for Contemporary Chinese Studies, Nottingham, 19-22 November.

LIEBER Marylène (2010) Chinese migrants in Switzerland: from mutual assistance to promoting economic interests, Journal of Chinese Overseas, 6 (1) [À paraître].

LI Minghuan (1998) Transnational links among the Chinese in Europe: a study of european-wide Chinese voluntary associations, in Gregor Benton and Frank Pieke, The Chinese in Europe, New York, St. Martin's Press.

LIVE Yu-Sion (1992) Les Chinois de Paris depuis le début du siècle. Présence urbaine et activités économiques, Revue européenne des migrations internationales, 8 (3), pp. 155-173.

LOUIE Andrea (2000) Re-territorializing transnationalism: Chinese American and the Chinese motherland, American ethnologist, 27 (3), pp. 645-669. 
LONGCHAMP Claude, AEBERSOLD Monica, ROUSSELOT Bianca et RATELBAND-PALY Sylvia (2005) Sans-papiers en Suisse : c'est le marché de l'emploi qui est déterminant, non pas la politique d'asile, Rapport final sur mandat de l'office fédéral des migrations, Berne, gfs.bern.

MA Laurence J.-C. (2003) Space, place, and transnationalism in the Chinese diaspora, in Laurence J.-C. Ma and Carolyn Cartier Eds., The Chinese diaspora. Space, place, mobility and identity, Boston, Rowman \& Littlefield.

MA MUNG Emmanuel (2002) Les mutations des migrations chinoises, VEI-enjeux : Nouvelles migrations, nouvelles formes de migrations, 131, pp. 129-145.

MEYER Jean-Baptiste et HERNANDEZ Valeria (2004) Les diasporas scientifiques et techniques : état des lieux, in Mihaela Nedelcu Éd., La mobilité internationale des compétences. Situations récentes, approches nouvelles, Paris, L'Harmattan.

NEDELCU Mihaela (Éd.) (2004) La mobilité internationale des compétences. Situations récentes, approches nouvelles, Paris, L'Harmattan.

NYIRI Pal (1999) Chinese organisation in Hungary, 1989-1996. A case study in PRC-oriented community politics overseas, in Frank Pieke and Hein Mallee Eds., Internal and international migration: Chinese perspectives, Richmond, Curzon.

NYIRI Pal (2004) Expatriating is patriotic? The discourse on new "migrants" in the People Republic of China and identity construction among recent migrant from PRC, in Brenda S.-A. Yeoh and Katie Willis Eds., State/Nation/Transnation. Perspectives on Transnationalism in the AsiaPacific, Londres et New-York, Routledge.

NYIRI Pal and SAVELIEV Igor R. (2002) Globalising Chinese Migration: Trends in Europe and Asia, Aldershot, Ashgate Publishing.

OCAC (2007) Statistical Yearbook of the Overseas Compatriot Affairs Commission, Taipei, OCAC.

PECORARO Marco et FIBBI Rosita (2008) Migrations chinoises en Suisse. Une analyse statistique, Textes du SFM 54, Neuchâtel, SFM.

PIEKE Frank (1992) Immigration et entreprenariat : les Chinois aux Pays-Bas, Revue européenne des migrations internationales, 8 (3), pp. 33-50.

PIEKE Frank, NYIRI Pal, THUNO Mette and CECCAGNO Antonella (Eds.) (2004) Transnational Chinese: Fujianese Migrants in Europe, Stanford, Stanford University Press.

PIEKE Frank (2007) Editorial Introduction: Community and Identity in the New Chinese Migration Order, Population, Space and Place, 13, pp. 81-94.

PIGUET Étienne (2004) L'immigration en Suisse. 50 ans d'ouverture, Lausanne, Presses polytechniques et universitaires romandes.

PINA-GUERASSIMOFF Carine (2003) Les nouveaux migrants chinois en Europe, Migrations et société, 15 (89), pp. 89-104.

ROCHA DA SILVA Pascal (2007) La population chinoise en Suisse à l'ère de la globalisation, mémoire de maîtrise en démographie économique et sociale, Université de Genève.

SKELDON Ronald (2004) China: from exceptional case to global participant, Migration Information Source.

SZTANKE Michaël (2005) Pékin - Paris : l'étudiant chinois est-il une marchandise ?, Hommes et migrations, 1254 , pp. 74-81.

THUNO Mette (2001) Reaching out and incorporating Chinese Overseas: The trans-territorial scope of the end of the 20th century, Chine Quarterly, 168, pp. 910-929.

VAUGHAN Robinson (1992) Une minorité invisible : les Chinois au Royaume-Uni, Revue européenne des migrations internationales, 8 (3), pp. 9-31.

WANG Gungwu (1992) The origins of Hua-Ch'iao, in Gungwu Wang, Community and Nation: China, Southeast Asia and Australia, St. Leonards, Allen and Unwin.

XIANG Biao (2003) Emigration from China: a sending country perspective, International Migration, 41 (3), pp. 21-48.

XU Bin (1956) L'économie des Chinois en Europe, Taipei, Oversea Édition [en chinois]. 
YAO Sun (1988) La situation des Chinois en Allemagne de l'Ouest, en Autriche et en Suisse, Taipei, Zhengzhong [en chinois].

ZHANG Guochu (2003) Migration of Highly Skilled Chinese to Europe: Trends and perpsective, International migration, 43 (3), pp. 73-97.

ZHOU Min and KIM Rebecca (2006) The Paradox of ethnicisation and assimilation, in Khun Eng Kuah-Pearce and Evelyn Hu-Dehart Eds., Voluntary association in the Chinese diaspora. HongKong, Hong-Kong University Press.

ZHOU Yu and CHIANG Lan-Hung Nora (2009) Assimilation and rising taiwanese identity: Taiwan-born immigrants in the United States, 1990-2000, Journal of Population Studies, 38, pp. 115-160. 